\title{
Shaping the GeV-spectra of bright blazars
}

\author{
Anita Reimer* \\ Institute for Theoretical Physics, University of Innsbruck, Austria \\ E-mail: anita.reimer@uibk.ac.at

\section{Lars Hunger} \\ Brainlinks-Braintools, University of Freiburg, Germany \\ E-mail: lars.hunger@brainlinks-braintools.uni-freiburg.de
}

\begin{abstract}
The non-thermal spectra of jetted Active Galactic Nuclei (AGN) show a variety of shapes and degrees of curvature in their low and high energy components. From some of the brightest FermiLAT blazars prominent spectral breaks at a few $\mathrm{GeV}$ have been regularly detected which is inconsistent with conventional cooling effects. We propose that the broad variety of spectral shapes including prominent breaks can be understood as an impact of injection modes.

We therefore present an injection model embedded in a leptonic blazar emission model for external Compton loss dominated jets of AGN which aims towards bridging jet emission with acceleration models using a phenomenological approach. Our model allows to consider the effects of continuous time-dependent injection of electrons into the jet with differing rates, durations, locations and power-law spectral indices. Here we show the impact of different injection rates and spectral indices on the ambient emitting particle spectrum observed at a given snapshot time. We found that varying these parameters has indeed notable influence on the spectral shapes, which in turn can be used to set interesting constraints on the particle injection scenario. We apply our model to the flare state spectral energy distribution of 3C 454.3 and PKS 1510-089 to constrain the required injection parameters. Our results indicate that impulsive-like particle injection is disfavored here. With this model we provide a basis for analyzing ambient electron spectra in terms of injection requirements, with implications for particle acceleration modes.
\end{abstract}

The 34th International Cosmic Ray Conference,

30 July- 6 August, 2015

The Hague, The Netherlands

\footnotetext{
* Speaker.
} 


\section{Introduction}

Blazars are jetted active galactic nuclei (AGN) that show a broad and often highly variable spectral energy distribution (SED) covering the whole electromagnetic band. Non-thermal relativistic particle populations in their jets are thought to be responsible for their variable continuum emission although the nature - electron-positron pairs or relativistic electron-proton populations is still under debate. The location within frequency space of the characteristic two-component SED is basis for the low-synchrotron peaked (LSP), intermediate-synchrotron peaked (ISP) and highsynchrotron peaked (HSP) blazar classification [2]. Bright flat-spectrum radio quasars (FSRQs), LSP- and occasionally ISP-type BL Lac objects show often significant spectral breaks or turn-over in the 1-10 GeV energy range, larger than expected from radiative cooling. So far proposed explanations include $\gamma \gamma$ - pair production in the broad-line region (BLR; e.g., [12], two-component $\gamma$-ray spectra [8] or corresponding breaks in the ambient particle distribution [1].

In this work we study, within the framework of an extern inverse Compton leptonic blazar emission model, the impact of various time-dependent injection scenarios of relativistic particles on the shape of the emitting particle spectrum, and photon spectrum. We focus in particular on the formation of strongly breaking ambient electron spectra. We apply our model to the observed broadband SEDs of 3C 454.3 and PKS 1510-089 which allows us then to draw inferences on the required particle injection scenario in those cases. A detailled description of the model and comprehensive parameter study is given in [9].

\section{The model}

Here we shall briefly summarize the main cornerstones of our model. We consider a standard leptonic blazar emission model where the non-thermal emission is originating from a magnetized (with field strength $B$ ) spherical symmetric emission region ("blob") of size $R_{b}$ (galaxy rest frame) that is moving with relativistic constant speed $\beta_{\Gamma} c$ along the AGN jet axis and viewed under a small angle. We consider the case where the dominant loss channel of the relativistic particles in the blob is inverse Compton (IC) scattering on external radiation fields such as radiation from the (assumed here Shakura Sunyaev) accretion disk and accretion disk radiation backscattered by the BLR. This case is particular suitable for FSRQs and LSP-type BL Lacs where those radiation fields are typically found particular luminous. For the BLR geometry we consider a spherical symmetric shell of gas between an inner $\left(R_{i}\right)$ and outer $\left(R_{o}\right)$ radius and a radially decreasing density gradient $n_{e} \propto R^{-\xi}$. We use a delta-function approximation for the BLR spectrum [13]. This model set-up follows broadly [6] and [7] except for the treatment of particle injection. Relativistic electrons following a power law with index $s$ between particle Lorentz factors $\gamma_{1}$ and $\gamma_{2}$ are continuously injected along the jet axis between heights $z_{a}$ and $z_{b}$ above the accretion disk with a time-dependent injection rate $\propto z^{-\alpha}$ (first proposed by [11]. The resulting emitting electron spectrum at height $z$ is then a superposition of the cooling electron spectra that have been injected and weighted by the corresponding injection rate. The resulting (snapshot) $\gamma$-ray spectra are then calculated from this ambient particle spectrum using the full Compton cross section with scattering in the head-on approximation. All further details can be found in [9]. 


\section{Results}

We present here selected results from our parameter study, with particular focus on the effect of injection rate and spectral index on the ambient particle spectrum; see [9] for further details.

Starting from a reference model with parameters $s=3, \alpha=2, z_{a}=200, z=z_{b}=2000, \gamma_{1}=$ $1000, \gamma_{2}=10^{6}$, Thomson optical depth of BLR gas $\tau_{\mathrm{BLR}}=0.01$ where $R_{i}=1000 R_{g}, R_{i}=1000 R_{g}$ ( $R_{g}$ is the gravitational radius) and $\xi=2$ we vary subsequently the values of $\gamma_{1}, s$ and $\alpha$ (Figs. 1-3).

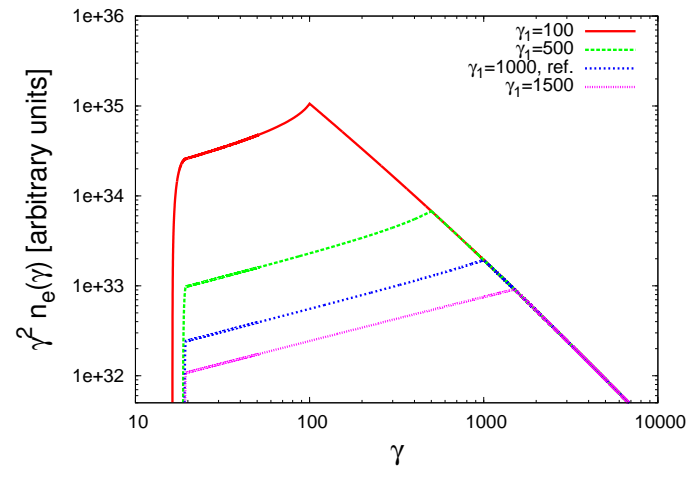

Figure 1: Emitting electron spectra for lower energy bound $\gamma_{1}$ of injected electron spectrum varying with respect to the reference model.

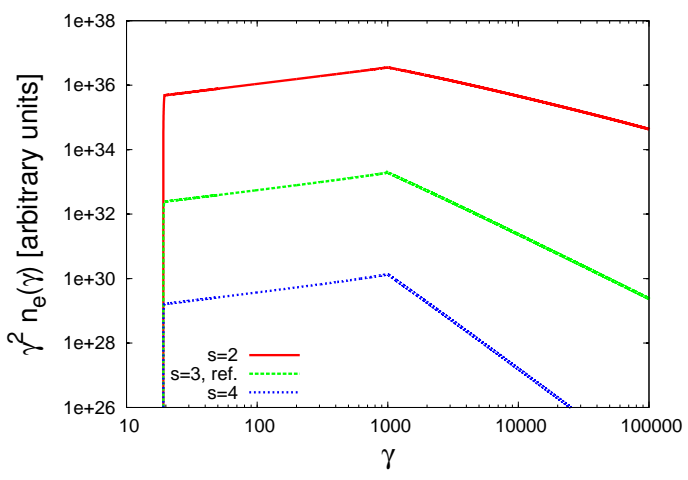

Figure 2: Emitting electron for spectral injection indices $s$ varying with respect to the reference model.

Fig. 1 shows that the position of the break in the ambient particle spectrum is in general determind by the lowest energy of the injected spectrum, $\gamma_{1}$. This is because below $\gamma_{1}$ only cooled electrons contribute to the emitting particle spectrum while above cooling and injection determines its shape. The injected spectrum and injection rate therefore influences the broadness of the break. Here the harder the injected spectrum the broader the break appears for a given injection rate (see Fig. 2). The injection rate then influences the shape of the emitting spetrum below the break. Here

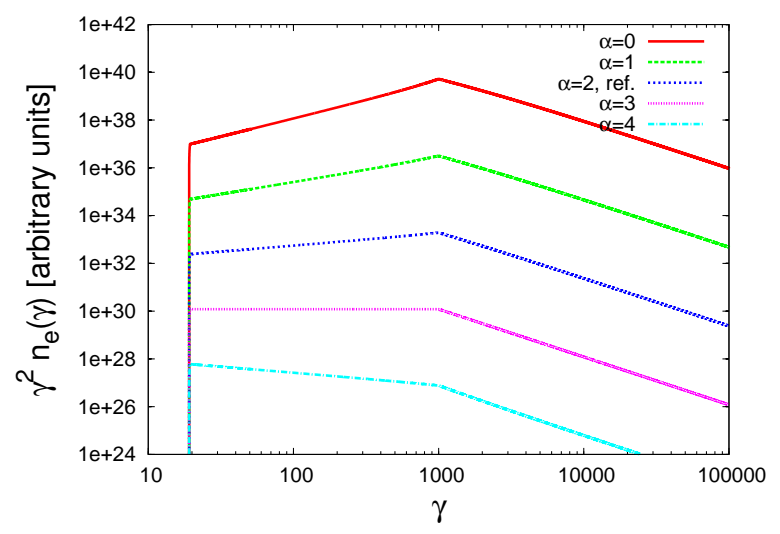

Figure 3: Ambient electron spectra with different injection rate parameters $\alpha$ with respect to the reference model. 
the lowest energies $\gamma \ll \gamma_{1}$ stem from particles injected at the lowest point $z=z_{a}$ where cooling rates are higher than at larger distances above the accretion disk. As a consequence very steep injection rate distributions result in softer ambient particle spectra below $\gamma=\gamma_{1}$ than shallower injection laws where very hard electron spectra can build up. This is depicted in Fig. 3.

The corresponding photon spectra from IC scattering in the accretion disk and at BLR reprocessed accretion disk radiation fields are displayed in [9]. We find a higher probability that the two components merge to one with increasing broadness of the resulting underlying particle spectrum.

\section{Applications}

In the following we apply our model to quasi-simultaneous broadband SEDs of two bright FSRQs, 3C 454.3 and PKS 1510-089. We focus in particular on their breaking GeV-spectrum up to $<10 \mathrm{GeV}$. This allows to neglect any possible absorption effects from propagation of $\gamma$-rays through the extragalactic background light as well as significant source internal absorption. We also neglect here any possible Synchrotron-Self Compton (SSC) contribution to the SED which typically may influence the $\mathrm{X}$-ray spectrum in a rather small energy range only.

\subsection{Modeling the SED of $3 C 454.3$}

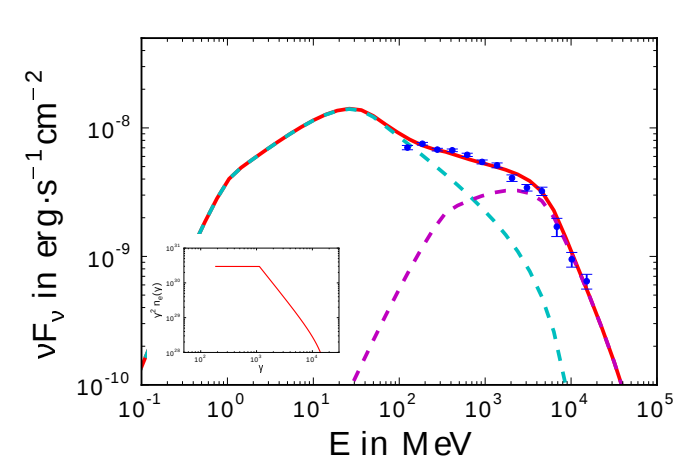

Figure 4: Modeling the $\gamma$-ray spectrum with Fermi-LAT data of the November 2010 flare [5] with the "2-component model"; inlet: the underlying emitting electron spectrum.

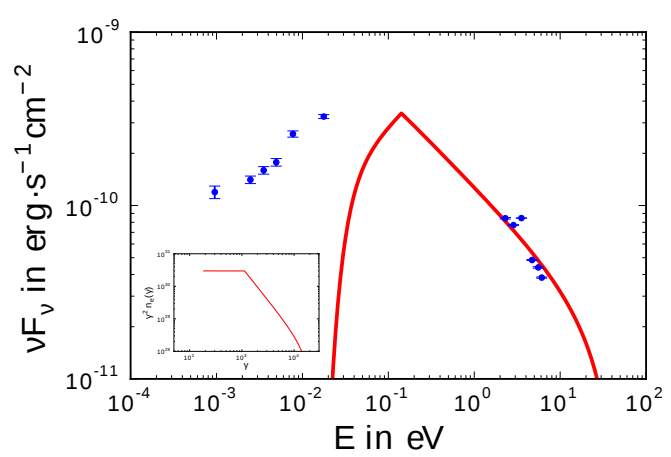

Figure 5: Corresponding model fits of the synchrotron spectrum of the November 2010 flare (data from [14] together with the underlying emitting electron spectrum (inlet).

We apply our model to the November 2010 flare state of FSRQ 3C 454.3 residing at redshift $\sim 0.859$ ([5], [14]). In the optically thin range the synchrotron spectra provide valuable constraints on the shape of the underlying particle distribution. We therefore include modelling the low energy hump of the SED (including synchrotron self-absorption) as well as the $\gamma$-ray spectra. We find two principally different model fits to explain the GeV-break in the $\gamma$-ray data: Either the break is due to the overlapping effect of the two IC components from BLR and accretion disk target photon fields (as has been proposed by [8]; see Figs. 4 \& 5; "2-component model"), or due to a break in the underlying electron distribution (see Figs. 6 \& 7; "BLR-dominated model"). The GeV-emission in the latter case is dominantly due to IC scattering in the BLR target radiation field. Other than in 


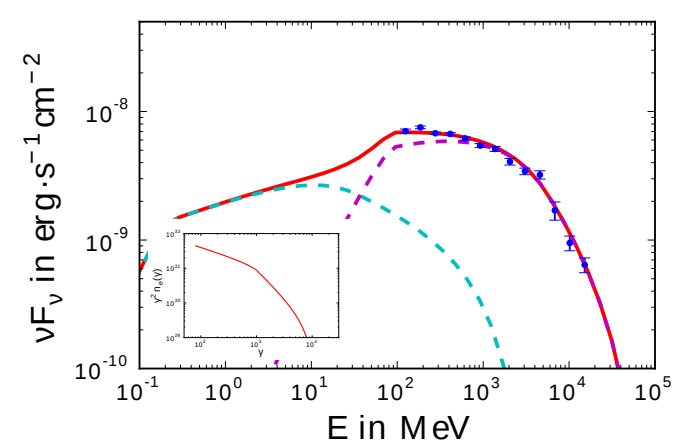

Figure 6: Modeling the $\gamma$-ray spectrum with Fermi-LAT data of the November 2010 flare [5] with the "BLR-dominated model"; inlet: the underlying emitting electron spectrum.

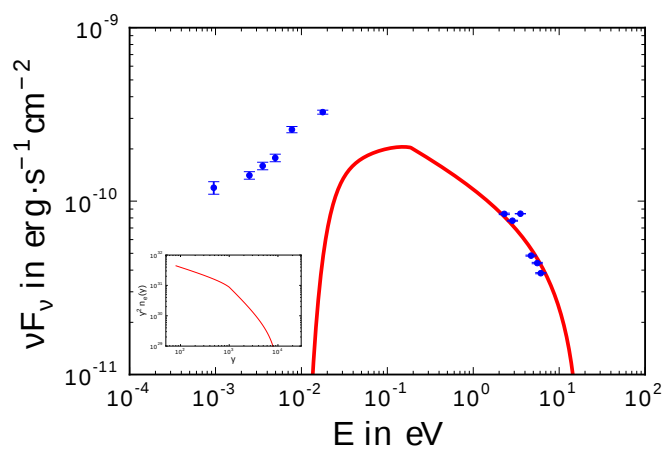

Figure 7: Corresponding model fits of the synchrotron spectrum of the November 2010 flare (data from [14] together with the underlying emitting electron spectrum (inlet).

[8] where the underlying particle distribution was treated with free parameters our model allows to determine the required injection details to fit the data.

For the 2-component model we found the injected spectral index to lie between $s=2$ and $s=3$ with the low energy cutoff in the range $\gamma_{1}=800 \ldots 1300$ and high energy cutoff $\gamma_{2}=(1 \ldots 2) \cdot 10^{4}$. Here we inject particles from heights $z_{a}=100 \ldots 800 R_{g}$ up to heights $z_{b}=1500 \ldots 3000 R_{g}$ into a magnetized region with magnetic field strength of $B=0.6 \mathrm{G}$. The emission region of size $R_{b}=$ $10^{16} \mathrm{~cm}$ is moving with bulk Lorentz factor $\Gamma=20$ (Doppler factor $\mathrm{D}=30$ ) along the jet axis. For the accretion disk luminosity we use 0.04 times the Eddington luminosity assuming a black hole mass of $2 \cdot 10^{9} M_{\odot}$. The relevant BLR parameters for our modelling are: $R_{i}=50 R_{g}, R_{o}=10^{4} R_{g}$, $\xi=2$ and $\tau_{\mathrm{BLR}}=0.008$. For the model fits we require injection rates with indices $\alpha \leq 4$. This indicates that instantenous-like injection modes do not represent the data well here.

The BLR-dominated model requires a higher Thomson optical thickness of the BLR $\tau_{\mathrm{BLR}} \approx$ 0.05 while all other BLR relevant parameters remain unchanged. We also keep the accretion disk luminosity as well as the speed of the moving "blob" of size $R_{b} \cdot 10^{15} \mathrm{~cm}$ at approximately the same value. Particles are injected between $z_{a}<200 R_{g}$ and $z_{b}=2000 \ldots .3000$ with power law indices in the range $s=1.5 \ldots .2 .5$ into a magnetized blob with field strength $B=1 \mathrm{G} . \gamma_{1}$ and $\gamma_{2}$ are similarily constrained as in the 2-component model. The injection rate index is here rather tightly constrained to lie in the range $\alpha=4 \ldots .4 .5$.

Hence, both model fits indicate that impulsive-like injection is unfavored in the framework of the here presented leptonic blazar emission model.

\subsection{Modeling the SED of PKS 1510-089}

The FSRQ PKS 1510-089 at redshift $\sim 0.361$ is also among the best observed $\gamma$-ray blazars with a well-constrained SED from radio to $\gamma$ rays. We apply here our model to the quasi-simultanuous broadband data from the January 2009 flare as published in [3], the first flare in a series of outbursts from this source in early 2009. These observations indicate variability of time scales of a day down to a few hours (e.g., [4]). The appearance of two new knots on VLBA $43 \mathrm{GHz}$ radio maps around 


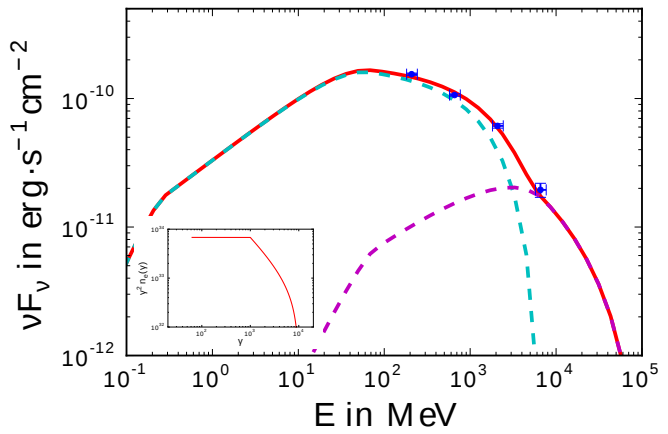

Figure 8: Modeling the $\gamma$-ray spectrum with Fermi-LAT data of the January 2009 flare [3] with the "2-component model"; inlet: the underlying emitting electron spectrum.

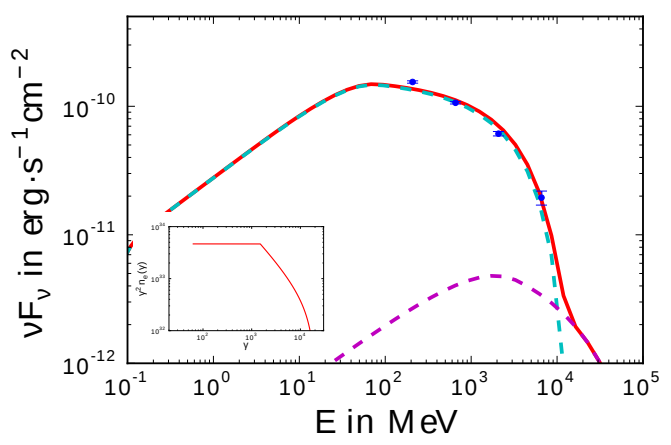

Figure 10: Modeling the $\gamma$-ray spectrum with Fermi-LAT data of the January 2009 flare [3] with the "accretion disk dominated model"; inlet: the underlying emitting electron spectrum.

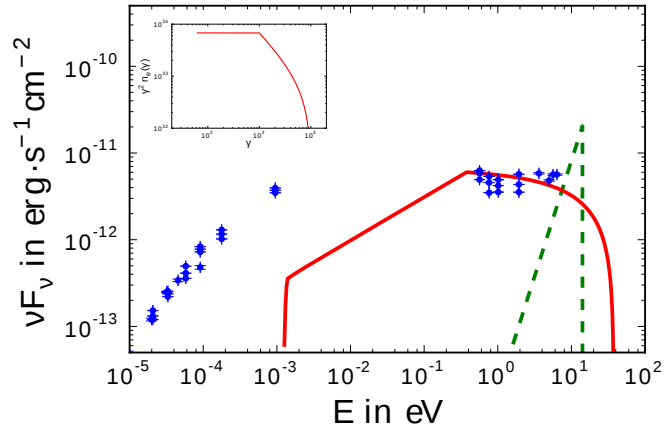

Figure 9: Corresponding model fits of the synchrotron spectrum of the January 2009 flare (data from [3]) together with the underlying emitting electron spectrum (inlet).

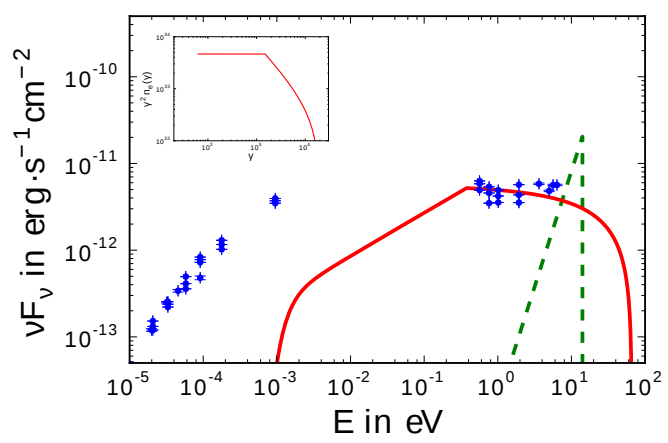

Figure 11: Corresponding model fits of the synchrotron spectrum of the January 2009 flare (data from [3]) together with the underlying emitting electron spectrum (inlet).

August 2008 and April 2009 that move with superluminal speeds of $22 c$ and $\sim 24 c$ along the jet seem to indicate a relation between these flaring events and their appearance [10]. The Fermi-LAT spectrum during the January 2009 flare indicates a pronounced decline at a few $\mathrm{GeV}$, similar as in the case of $3 \mathrm{C} 454.3$.

The results of our modeling indicate again the possibility to interpret this GeV-break as either due to the composite effect of the two IC-components (with accretion disk and BLR as target radiation fields; "2-component model") or due to an intrinsic break in the emitting particle distribution resulting from continuous time-dependent particle injection. Here the photon spectrum is dominated by IC scattering in the accretion disk radiation field ("accretion disk dominated model").

The model represention of the data using the 2-component model yielded quite similar parameter values as in the case of 3 C 454.3. Figs. 8 \& 9 show the model fits with the following parameter values: injected particle spectrum with spectral index $s=2$ within the energy range $\gamma_{1}=1000$ and 
$\gamma_{2}=10^{4}$; particle injection from heights $z_{a}=200 R_{g}$ up to heights $z_{b}=2000 R_{g}$ with injection rates parametrized with index $\alpha \leq 3$ into a region with magnetic field strength $B=1.5 \mathrm{G}$; the emission region is of size $R_{b}=10^{15} \mathrm{~cm}$ and moving with bulk Lorentz factor $\Gamma=20$ (Doppler factor $\mathrm{D}=30$ ) along the jet axis. For the accretion disk luminosity we use 0.04 times the Eddington luminosity assuming a black hole mass of $2 \cdot 10^{9} M_{\odot}$. The BLR parameters used for our modelling are $R_{i}=50 R_{g}$, $R_{o}=10^{4} R_{g}, \xi=2$ and $\tau_{\mathrm{BLR}}=0.005$. Hence here particle injection starts and ends deeply within the BLR.

In the case of fitting the data with the accretion disk dominated model we require particle injection starting at $z_{a}=200 R_{g}$ and ending at $z_{b}=2000 R_{g}$ at essentially the inner $\left(R_{i}=100 R_{g}\right)$ and outer $\operatorname{rim}\left(R_{o}=2000 R_{g}\right)$, respectively, of the BLR with $\tau_{\mathrm{BLR}}=0.002$. The fitting parameters used for the model representations shown in Figs. $10 \& 11$ are otherwise rather similar to the 2component model case: $s=2, \gamma_{1}=1500, \gamma_{2}=2 \cdot 10^{4}, \alpha \leq 3, \Gamma=20, D=30$ and unchanged accretion disk radiation parameters.

We note that in both model fits presented here the values of the particle injection rates required indicate again that impulsive-like injection is disfavored.

\section{Summary}

We considered the case of continuous and time-dependent particle injection (parametrized by powerl-law injection rates) into an emission region that is moving with relativisic speed along the jet within the framework of an external Compton blazar emission model with accretion disk and BLR target photon fields. Such setup is suitable for strong-line blazars such as FSRQs. We find that a broad range of spectral shapes of the emitting particle distribution, and correspondingly photon spectra, can be explained. In particular spectral breaks that are larger than expected from cooling are found. Our simple phenomenological approach in terms of particle energization has the potential to set constraints on the required particle acceleration mechanism when applied to blazar spectral data. We demonstrated this by applying our model to the flare states of 3C 454.3 and PKS 1510-089 which show pronounced breaks at GeV energies. For both sources we were able to explain these breaks by either the composite effect of Compton scattered BLR and accretion disk photons (confirming the suggestion by [8]), or by a one-component IC model whith an intrinsic break in the ambient particle distribution built up from continuous particle injection and cooling, while complying with the broadband data as well. The required particle injection parameters in all cases disfavor impulsive-like particle injection but point towards a scenario where fairly narrow power-law particle spectra are continuously and with a gradually decreasig rate injected into the moving plasma blob.

\section{Acknowledgement}

This work was performed partly on the computational resource bwUniCluster funded by the Ministry of Science, Research and the Arts Baden-Württemberg and the Universities of the State of Baden-Württemberg, Germany, within the framework program bwHPC. 


\section{References}

[1] A.A. Abdo, et al., Spectral Properties of Bright Fermi-Detected Blazars in the Gamma-Ray Band, ApJ 710 (2010a) 1271

[2] A. A. Abdo, et al. The First Catalog of Active Galactic Nuclei Detected by the Fermi Large Area Telescope, ApJ 715 (2010b), 429

[3] A.A. Abdo, et al., The Spectral Energy Distribution of Fermi Bright Blazars, ApJ 716 (2010c) 30

[4] A.A. Abdo, et al., Fermi Large Area Telescope and Multi-wavelength Observations of the Flaring Activity of PKS 1510-089 between 2008 September and 2009 June, ApJ 721 (2010d) 1425

[5] A.A. Abdo, et al., Fermi Gamma-ray Space Telescope Observations of the Gamma-ray Outburst from 3C454.3 in November 2010, ApJ 733 (2011) L26

[6] C.D. Dermer \& R. Schlickeiser, Model for the High-Energy Emission from Blazars, ApJ 416 (1993) 458

[7] C.D. Dermer, J.D. Finke, H. Krug \& M. Böttcher, Gamma-Ray Studies of Blazars: Synchro-Compton Analysis of Flat Spectrum Radio Quasars, ApJ 692 (2009) 32

[8] J.D. Finke \& C.D. Dermer, On the Break in the Fermi-Large Area Telescope Spectrum of 3C 454.3, ApJL 714 (2010) L303

[9] L. Hunger \& A. Reimer, Shaping the GeV-spectra of birght blazars, A\&A submitted (2015)

[10] A. P. Marscher, et al., Probing the Inner Jet of the Quasar PKS 1510-089 with Multi-Waveband Monitoring During Strong Gamma-Ray Activity, ApJ 710 (2010) L126

[11] A. Mücke \& M. Pohl, The contribution of unresolved radio-loud AGN to the extragalactic diffuse gamma-ray background, MNRAS 312 (2000) 177

[12] J. Poutanen \& B. Stern, GeV Breaks in Blazars as a Result of Gamma-ray Absorption Within the Broad-line Region, ApJL 717 (2010) L118

[13] F. Tavecchio \& G. Ghisellini, The spectrum of the broad-line region and the high-energy emission of powerful blazars, MNRAS 386 (2008) 945

[14] A. Wehrle et al., Multiwavelength Variations of 3C 454.3 during the 2010 November to 2011 January Outburst, ApJ 758 (2012) 72 